\title{
Vibration and Modal Analysis of Low Earth Orbit Satellite
}

\author{
Asif Israr \\ Department of Mechanical Engineering, Institute of Space Technology, 1 Islamabad Highway, Islamabad 44000, Pakistan
}

Correspondence should be addressed to Asif Israr; asifisrar@yahoo.com

Received 28 February 2014; Revised 6 June 2014; Accepted 8 June 2014; Published 3 August 2014

Academic Editor: Longjun Dong

Copyright (C) 2014 Asif Israr. This is an open access article distributed under the Creative Commons Attribution License, which permits unrestricted use, distribution, and reproduction in any medium, provided the original work is properly cited.

\begin{abstract}
This paper presents design, modeling, and analysis of satellite model used for remote sensing. A detailed study is carried out for the design and modeling of the satellite structure focusing on the factors such as the selection of material, optimization of shape and geometry, and accommodation of different subsystems and payload. The center of mass is required to be kept within the range of (12) $\mathrm{cm}$ from its geometric center. Once the model is finalized it is required to be analyzed by the use of Ansys, a tool for finite element analysis (FEA) under given loading and boundary conditions. Static, modal, and harmonic analyses in Ansys are performed at the time of ground testing and launching phase. The finite element analysis results are also validated and compared with the theoretical predictions. These analyses are quite helpful and suggest that the satellite structure does not fail and retains its structural integrity during launch environment.
\end{abstract}

\section{Introduction}

CubeSats are minuscule satellites designed for low earth orbit (LEO) with a purpose to use universities worldwide for space research and exploration. Usually, these satellites have a mass of approximately $1.33 \mathrm{~kg}$, have a volume of $10 \mathrm{~cm}$ cube, and use standard modules for their integrated circuits. California Polytechnic State University (Cal Poly) and Stanford University contributed initially towards development of CubeSats. A CubeSat of $(10 \times 10 \times 10) \mathrm{cm}$ is termed as CubeSat $1 \mathrm{U}$ (one unit) and extends in one direction only. Similarly, CubeSats $2 \mathrm{U}$ and $3 \mathrm{U}$ have also been built and launched for a specific mission. In addition, CubeSats $12 \mathrm{U}$ have now been proposed to cover more complex science and defense goals rather than limiting their applications in academia. Launch and deployment of these CubeSats are usually made through a common deployment system developed by Cal Poly and named Poly-PicoSatellite Orbital Deployer (P-POD). There are several types of CubeSats that have been developed and deployed for specific mission such as research and development satellites, earth remote sensing satellites, and space tethers satellites. Typical examples of earth remote sensing CubeSats are Quakesat, SwissCube, PLUME, and Firefly, and so forth. Details of these CubeSats are available in the literature.
LEO satellites are commonly used for communication and earth imaging enclosed by a payload of signal processing module only. In designing of LEO satellite, the model is premeditated by an appropriate selection of material based on low weight, high strength to weight ratio, and space qualified. In addition, basic geometry of the satellite body is also defined by considering the factors such as heat distribution and heat dissipation, weight of fasteners, accessibility and maintainability, accommodation of subsystems, center of gravity, and manufacturing cost. In the existing literature, it is found that aluminum alloy can be selected as a material and structure is made of cubical shape because of the reasons mentioned above. SwissCube [1] structure described a better representation for design and selection of the material for the satellite construction. The monoblock can be introduced that acts as a back bone for the construction of the basic geometry of the satellite structure as reported in the SwissCube [1] and SATAX Project [2]. It helps to increase the strength of the whole structure and mainly performs three different tasks: proper aligning of the geometry, holding of all the attitude thrusters, and assembling of subparts on it. In this way a very strong, robust, and extremely light frame structure can be obtained. Sedighi and Mohammadi [3] performed static and dynamic analysis of small satellite. They maintained a safety factor higher than five for the analysis by increasing or 
decreasing stringer height and increasing trays and stringer thickness. The measured first natural frequency was quite below the launcher requirements. They tried to shift the natural frequency to higher value by the increase of trays and stringer thickness but no avail. However, with increasing stringer height to $20 \mathrm{~mm}$ it considerably had an effect on frequencies. In addition, it was also observed that without stringer the first natural frequency dropped significantly. In modal analysis the first and fifth natural frequency at constant stringer height of $6 \mathrm{~mm}$ were $41.5 \mathrm{~Hz}$ and $100.4 \mathrm{~Hz}$, respectively, at a tray thickness of $2 \mathrm{~mm}$ in longitudinal direction; similarly $41.8 \mathrm{~Hz}$ and $101.8 \mathrm{~Hz}$, respectively, were observed in the lateral direction. With the increase of tray thickness to $10 \mathrm{~mm}$, the first natural frequency increased both in longitudinal and lateral directions. RASAT satellite [4] described the analysis carried out on the main stiffened and honeycomb panel. Normal mode and stress analysis were performed by the use of Ansys. In normal mode analysis the structure was constrained at the place of bolts and the first frequency was $86 \mathrm{~Hz}$. In stress analysis static inertial force of $60 \mathrm{~g}$ was applied in all three directions of the structure and the maximum local stress was $161 \mathrm{MPa}$ which was far less than the strength of the material used; hence the structure was safe under these conditions. Fufa et al. [5] investigated the locking processes of satellite and deployment interaction. The modal analysis of satellite solar panels was performed and results were illustrated with extremely good agreement. Libin and Hui [6] carried out the analysis on the telescopic frame using Ansys. Solid 95 was used as an element type for the structural analysis as this element type ignores most of the irregular shapes of the structure. Furthermore, the bolts of the holes were ignored as these were relatively very small. The analysis showed that the maximum stress and deformation were greater than the allowable design limit. A centric brace was introduced in the structure which drastically reduced the deformation in the structure. The model analysis was also carried out at different load steps and observed variation in their frequencies. Cihan et al. [7] used Ansys to analyze the behaviour of the structure by employing different element types, meshing type of quadrilaterals for shell and hexahedral for volumes. In static analysis the maximum von Mises stress was $5.0956 \mathrm{MPa}$ at the intersection points which was less than the yield strength of aluminum and the displacement of $0.004 \mathrm{~m}$ was observed on the top panel. In model analysis the first and last natural frequency were $633.25 \mathrm{~Hz}$ and $1948.3 \mathrm{~Hz}$, respectively; the last frequency was extremely high but still it is within the range of launch frequency. Static analysis of the AdeSat was performed by Braegen et al. [8]. AdeSat consists of four square rails and six flat plates. The top solar panel was simulated by placing a pressure caused by the load of the solar cells, and the deployable panels were treated similarly with their load acting onto the side plates. In Ansys, the PCBs were considered point masses. For the validation of satellite subsystems additional stress analyses were also carried out for the PCBs, hinge, hinge pin, and bolts. These analyses were performed using Ansys under given frequency range. Murtaza [9] presented a small satellite design of the weight of $55 \mathrm{~kg}$ using COTS for low earth orbit and remote sensing applications. All the subsystems are designed along with budget requirement of power, mass, and dimension with necessary calculation and showed that it satisfies all the design requirements.

Satellite laser ranging (SLR) is a useful technique to determine the geocentric position of the retroreflector equipped satellite using laser pulses back to the transmitting site. The satellites used for IERS (International Earth Rotation and Reference Systems Service) applications are known as LAGEOS-1 and LAGEOS-2 which were launched in 1976 and 1992, respectively, with an altitude of $5900 \mathrm{~km}$ but in different orbital planes. They both used fused silica glass cube-corner retroreflectors. The range to the LAGEOS satellites is now easily measured from a single laser pulse with a precision of less than $1 \mathrm{~cm}$. It is pertinent to mention that SLR is sensitive to the time history of its motion with respect to the origin of the terrestrial frame of reference and to the location of the earth's centre of mass. Scientists and engineers can find a lot of literature on this subject; however, few papers are presented here to give readers an idea of how this technique can be beneficial. Tapley et al. [10] discussed the SLR measurement strategies particularly its capabilities for estimation of geophysical parameter, that is, investigation of geodynamics and oceanography and exact orbit determination. The detailed analysis approach followed by NASA Crust Dynamic Project is provided and recent improvements in LAGEOS laser ranging data in terms of its quality and geophysical distribution have been reviewed. It is concluded that SLR system provides accurate measurements for orienting the earth within a quasiinertial reference system and deployment of additional and improved laser ranging systems will expand ground based tracking network. Iorio [11] developed an alternative approach to calculate lense thrilling effect on Keplerian orbital elements of a test particle falling freely in the field of different kinds of axially symmetric central sources. For perfectly spherical source the lense thrilling precessional rates for the node and the perigee were obtained. Departure from sphericity of the gravitational source showed effect on the node and perigee through additional secular rates. Iorio [12] further attempt was to measure the uncertainties in lense thrilling effect with the application of SLR technique to the existing LAGEOS and LAGEOS-II terrestrial satellite and LARES spacecraft. Different approaches were followed to extract Lense thrilling signal from data of LAGEOS type satellites by including gravitomagnetic force into the dynamic force. It was concluded that accuracy is disturbed by nongravitational perturbations induced in the satellite when it is orbited at lower altitudes. Renzetti [13] examined the low-altitude effects of LARES to determine their impact on the outcome of the hoped $1 \%$ frame-dragging measurement in the LARES-LAGEOS experiment. GIF48 model was used. The analysis showed that spherical harmonics of the earth gravity field with degree $l>$ 60 may represent a threat and their errors map significantly into LARES orbital disturbances compared to frame dragging because for $l>60$ the uncertainties in the LARES node precession get larger. Renzetti [14] further presented the history of complex, intricate, and branched attempts made by several researchers to measure lense thrilling effects in the terrestrial gravitational field with artificial satellites. Tests were conducted with different combinations of satellites, that 
is, LAGEOS, perigee of LAGEOS-2, CHAMP, and GRACE models. The use of LAGEOS and LAGEOS-2 provided the most promising way to detect the orbital lense thrilling drag in earth's surrounding. The addition of other SLR satellites such as Starlette and Stella has shown to enhance perturbations by a wide range of even zonal multipoles of terrestrial gravitational field because of their mounting at lower altitude as compared to LAGEOS and LAGEOS-2. Degnan [15] presented a review about the recent advancements in the field of SLR, mathematical formulation for computation of signal strength, single- and two-color techniques for direct measurement of refraction and atmospheric delay. An overview of retroreflector theory is also given which provides simple analytical model for computation of satellite target cross-section and impulse response. It is concluded that high altitude satellites because of their marginal cross-section broaden the return pulse and larger diameter satellites can be developed to improve two-color performance.

Based on the overview of SLR presented above, it will be quite interesting to conduct a separate study for analyzing the retroreflector frame that should meet the request on intensity, rigidity, security, and so forth. It will further improve the precision and functionality of the satellite retroreflector equipped satellite keeping into consideration the minimal distortion under different loads such as axial inertia and radial inertia during machining, assembling, and satellite shot process, and at present it is beyond the scope of this study.

In the subsequent sections a simple elliptical orbit configuration is presented to get insight of elliptical orbit and defines some of the important parameters such as orbital position, eccentricity, and semimajor axis. During satellite launch, satellite is subjected to various external loads resulting from vibroacoustic noise, booster ignition and burn out, propulsion system engine vibration, steady-state booster acceleration, and much more. Hence, design, model, and analyses are performed to device such a structure that should meet the launcher requirements followed by results and conclusions.

\section{Simple Elliptical Orbit Configurations}

A low earth orbit is an economical solution for the placement of satellites and offers low communication time delay and high bandwidth. LEO is an orbit followed around earth with an altitude of $160 \mathrm{~km}$ and $2,000 \mathrm{~km}$ and an orbital period of $88 \mathrm{~min}$ and $127 \mathrm{~min}$, respectively. Below $160 \mathrm{~km}$ approximately the objects experience altitude loss and orbital decay. As known, artificial satellites are positioned in LEO or geocentric orbit using geocentric coordinate systems. Geocentric coordinates are an earth centered coordinate system, $C$ as the origin of placing objects. Figure 1(a) explains the elliptical orbit and defines some of the important parameters such as orbital position, $r$; it identifies the satellite instantaneous location eccentricity, $e$, and major axis midpoint $Q$ of length $2 a$, and $a$ is the semimajor axis. The satellite's motion can be defined by orbital elements right ascension, $\Omega$, orbit inclination, $i$, argument of perigee, $\omega$, eccentricity, $e$, semimajor axis, $a$, and time across ascending node, $t$, and they all determine the position of satellite in the space completely as shown in Figure 1(b). Once the subsatellite path meets the equatorial plane the ascending node $N$ location can be identified. The right ascension, $\Omega$, of the ascending node measures angle from Aries direction to the ascending node $N$ eastward. The orbit's inclination, $i$, defines the angle of the equatorial plane and the satellite orbital plane. The argument of perigee, $\omega$, defines the angle of the point of perigee $P$ and the ascending node $N$ in the satellite's orbital plane.

\section{Vibration and Modal Analysis}

In this study, satellite structure is designed and modeled subsystems to acquire the engineering qualified model. The constructed model is required to meet all the testing parameters under given loading conditions. Various aspects are considered in designing the structure such as strength, life, material, and shape. Aluminum alloy 7075-T6 is used here for building the basic structure of the system. This material is light in weight and has high stiffness to withstand loading conditions. Although there are many shapes of satellite structure such as cubical, hexagonal, and circular that are being currently used in space industry, these shapes can be employed according to application, mission orbit, and internal space requirement. A cubical configuration is selected with external dimension of $(1140 \times 1190 \times 1569) \mathrm{mm}$ and weighs $650 \mathrm{~kg}$, excluding the solar panels. In order to keep the structure aligned and stable, a monoblock is introduced that provides more strength to the structure. With the addition of monoblock the weight of the structure is increased; however, there must be a compromise in designing; thus strength over weight is more important in this case. Mass distribution is of utmost importance for modeling the subsystems into the inner structure of the satellite. These subsystems are placed in such a manner to keep the center of gravity (CG) of the entire structure within $(1-2) \mathrm{cm}$ from the geometric center. Otherwise, a catastrophic failure may occur if this safe range is not achieved. For these reasons two heavy subsystems, that is, batteries and two telescopes, are placed above and below the cubic structure walls, respectively. The remaining subsystems are arranged accordingly. $5 \mathrm{~mm}$ distance is also maintained between all subsystems to avoid heat dissipation among these subsystems.

The complete satellite structure is modeled in design software. The CG of the cubical body is then calculated using some basic formulations and found to lie in the specified range as mentioned above. The built satellite structure is then exported in Ansys. In Ansys the structure is assembled and meshed successfully for analyzing the structural body of the system. Static, modal, and harmonic analyses are performed to analyze the integrity of the structure under given loading conditions. The main objectives of these analyses are to ensure that the satellite structure survives during launch loads. For these analyses, it is assumed that the satellite is constrained in space with the launch vehicle interface as in actual practice it is constrained by the use of some basic mechanism. Furthermore, these analyses are simplified by assuming that screwing of the subsystems is omitted, and all 


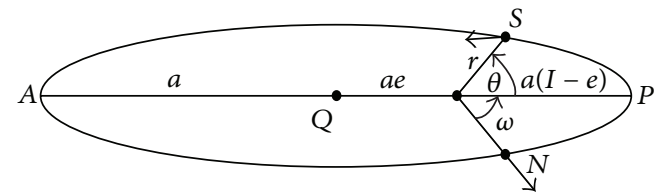

(a) Parameters of elliptical orbit

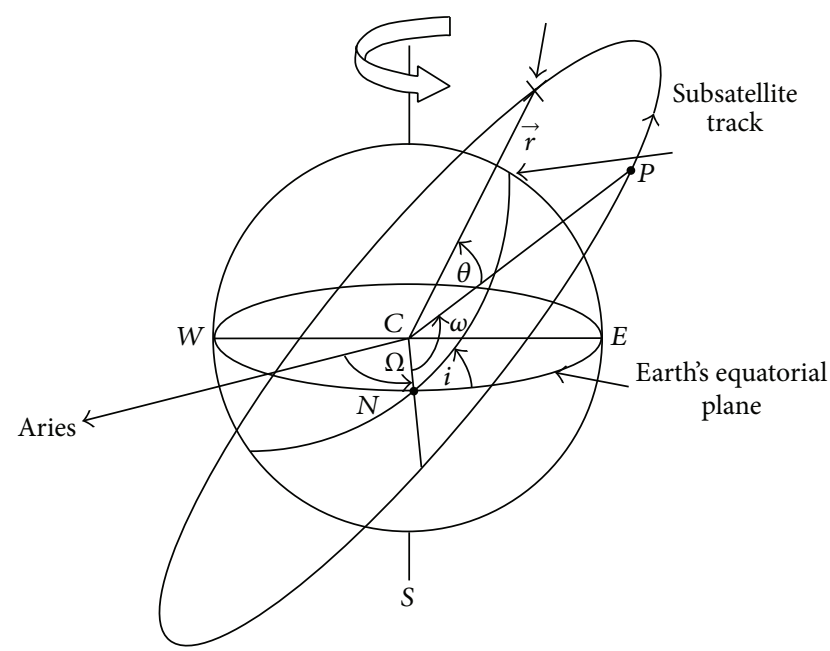

(b) Determination of satellite in the space

FIGURE 1: Elliptical orbit configurations.

extra holes on the structure for various reasons are neglected as they have very small dimensions.

The results obtained from the FE analysis can also be verified by simple formulation for first natural frequency only. There is no such relation available in the literature for the vibration analysis of the satellite structure. For instance, a beam can best describe the present configuration. The theoretical natural frequency of the beam with one end fixed and the other end free for the first frequency mode is given by

$$
f_{1}=\frac{1.875^{2}}{2 \pi} \sqrt{\frac{E I}{m L^{4}}},
$$

where $L$ is the length of the beam, $I$ is the area moment of inertia, $E$ is the modulus of elasticity, and $m$ is the mass of the beam.

Now, static, modal, and harmonic analyses are performed in the subsequent sections.

3.1. Static Analysis. Static analysis is used to estimate the stresses, strains, displacements, and forces in the structural components of the system. Hence these analyses are essential to measure the strength of the satellite structure. Generally, steady loading and response conditions are assumed during the analysis. In this analysis, the weight of the entire satellite and a force of magnitude $9 \mathrm{~g}$ are acted on the geometric center of the structure while the lower legs of the base are fixed. The maximum deformation and stresses are found at the top sheet and can be seen in Figure 2.

The maximum deformation $\left(1.63 \times 10^{-4} \mathrm{~m}\right)$ is far less as compared to the dimensions of the structure; similarly, the maximum equivalent stress is $3.415 \times 10^{6} \mathrm{~Pa}$ which is also lower than the yield strength of aluminum. It means structure can sustain the loading conditions, does not fail, and maintains its integrity during actual launch after the application of maximum static load.
3.2. Modal Analysis. As per global stiffness requirements, the fundamental frequency of the entire system must be $\geq 30 \mathrm{~Hz}$. By doing so, the structure will not resonate and will survive during launch without any deformation. In order to get the frequencies and mode shapes of the satellite structure, modal analysis needs to be carried out for dynamic loading conditions. Solid186 is used as an element type for examining the satellite structure. Table 1 shows ten modal frequencies and deflection values against each frequency value.

The first modal frequency can also be validated as obtained from modal analysis from the theoretical relation as described in (1). It can be seen that there is a little difference between the two results, and element type solid186 is the right choice for such analysis. The modal shapes are also illustrated in Figure 3.

3.3. Harmonic Analysis. The harmonic analysis is also performed to determine the maximum possible stress on the structure. It is usually done for the vertical alignment of the satellite. The qualification loading levels as shown in Table 2 are used here for analyzing the satellite structure.

Mode superposition method is applied for the solution in Ansys with a damping of $1 \%$. The maximum stresses and deflections are tabulated in Table 3. These can also be demonstrated in Figures 4 and 5. Within the frequency range as mentioned $(0-200) \mathrm{Hz}$, catastrophic failure does not observe in the satellite structure. Table 3 shows that the maximum harmonic stress for the structure is $8.50 \times 10^{8} \mathrm{~Pa}$ at the side sheet of the cubic structure in case of longitudinal direction. Similarly, the maximum stresses evaluated by the static analysis are $3.415 \times 10^{6} \mathrm{~Pa}$ at the top sheet. Therefore it does not exceed the yield strength of the material used.

\section{Conclusions}

The satellite structure and its associated subsystems are designed and modeled in such a manner to keep the center of 


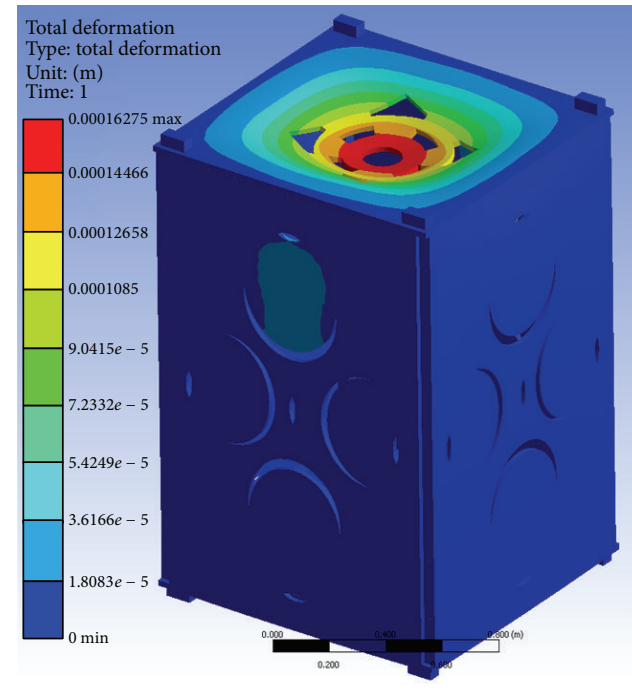

(a) Deformation pattern

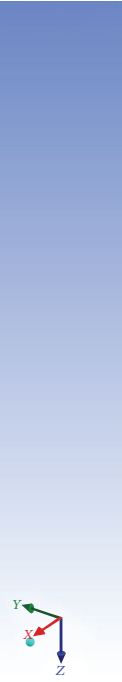

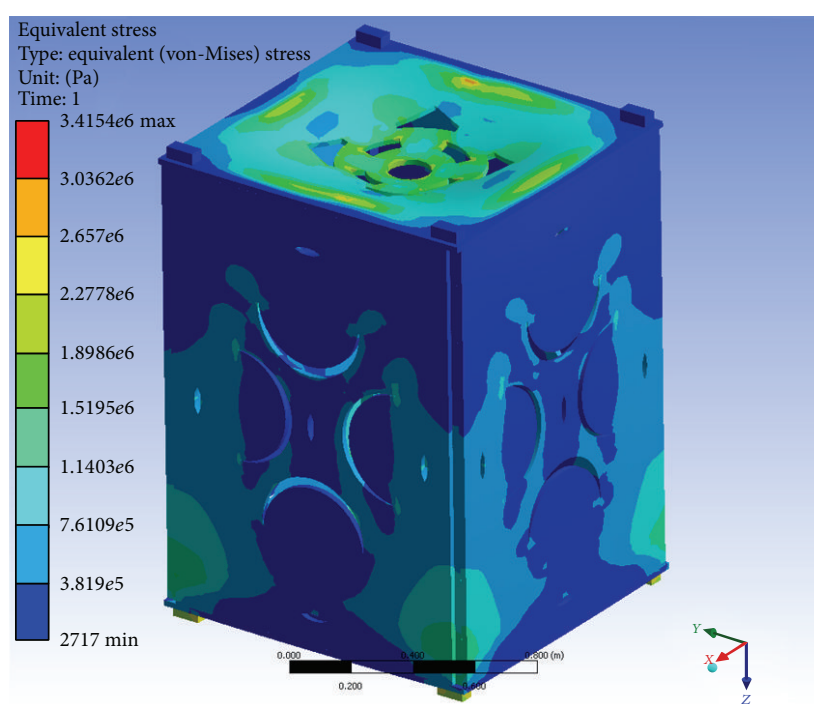

(b) Stress pattern

FigURE 2: Deformation and stress pattern.

TABLE 1: Ten modes of vibration.

\begin{tabular}{lcc}
\hline Modes & Frequency $[\mathrm{Hz}]$ & Def. $[\mathrm{m}]$ \\
\hline 1 & 82.03 & 0.2037 \\
2 & 89.58 & 0.2334 \\
3 & 97.82 & 0.2269 \\
4 & 114.07 & 0.1869 \\
5 & 137.51 & 0.1962 \\
6 & 141.97 & 0.2507 \\
7 & 143.13 & 0.2389 \\
8 & 146.88 & 0.2716 \\
9 & 151.31 & 0.2524 \\
10 & 157.43 & 0.2782 \\
\hline
\end{tabular}

TABLE 2: Qualification loading levels.

\begin{tabular}{lcc}
\hline & $\begin{array}{c}\text { Normal to the mounting } \\
\text { plane }\end{array}$ & $\begin{array}{c}\text { Parallel to the mounting } \\
\text { plane }\end{array}$ \\
\hline $\begin{array}{l}\text { Freq. }[\mathrm{Hz}] \\
0-200\end{array}$ & Levels & \\
\hline
\end{tabular}

mass within the range of $(1-2) \mathrm{cm}$ from the geometric center of the structure. A simpler model of monoblock is introduced for strengthening the entire structure. Accessibility of the subsystems is easier to handle in this case and the structure does not exceed the yield strength of the material used.

All these analyses are performed under given loading and boundary conditions. In static analysis, the maximum deformation and stress are found to be $1.63 \times 10^{-4} \mathrm{~m}$ and 3.415 $\mathrm{MPa}$, respectively, which are in the control limits when compared with the yield strength of the material (aluminum
TABLE 3: Harmonic analysis results.

\begin{tabular}{lcccc}
\hline & $\begin{array}{c}\text { Max. normal } \\
\text { stress }[\mathrm{Pa}]\end{array}$ & $\begin{array}{c}\text { Freq. } \\
{[\mathrm{Hz}]}\end{array}$ & $\begin{array}{c}\text { Max. def. } \\
{[\mathrm{m}]}\end{array}$ & Phase angle \\
\hline Longitudinal & $8.50 \times 10^{8}$ & 160 & $6.70 \times 10^{-2}$ & $29^{\circ}$ \\
Lateral & $2.76 \times 10^{8}$ & 80 & $4.91 \times 10^{-2}$ & $20^{\circ}$ \\
\hline
\end{tabular}

alloy 7075-T6 here). In modal analysis, mode shapes can easily be seen at the outer surfaces of the structure. A maximum displacement of $0.2782 \mathrm{~m}$ is observed at the side sheet of the cubic body for a frequency of $157.43 \mathrm{~Hz}$. Similarly, harmonic analysis is performed for a frequency range of $(0-$ 200) $\mathrm{Hz}$ with the application of two forces $8 \mathrm{~g}$ parallel to the mounting plane and $12 \mathrm{~g}$ normal to the mounting plane. The amplitudes at lateral and longitudinal directions are found to be $6.70 \times 10^{-2} \mathrm{~m}$ at a frequency of $160 \mathrm{~Hz}$ and $4.91 \times 10^{-2} \mathrm{~m}$ at a frequency of $80 \mathrm{~Hz}$, respectively, which is very small under given loading and boundary conditions.

In summary, the satellite structure is modeled and analyzed for static, modal, and harmonic response to ensure that the structure sustains in the harsh launch loads. The obtained estimate values of maximum stresses and deflections are less than the failure values. A brief overview of retroreflector equipped satellite for SLR is also presented and proposed that a separate study is required initially for the retroreflector frame to be included to improve the performance of the entire cubic structure.

\section{Conflict of Interests}

The author declares that there is no conflict of interests regarding the publication of this paper. 


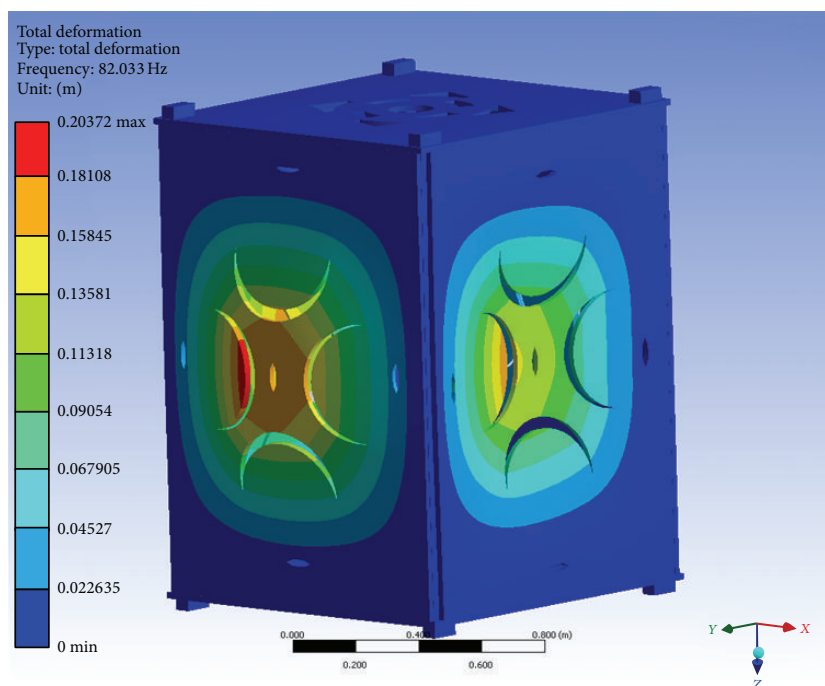

(a) Mode 1: Freq. $=82.03 \mathrm{~Hz}$ and Def. $=0.2037 \mathrm{~m}$

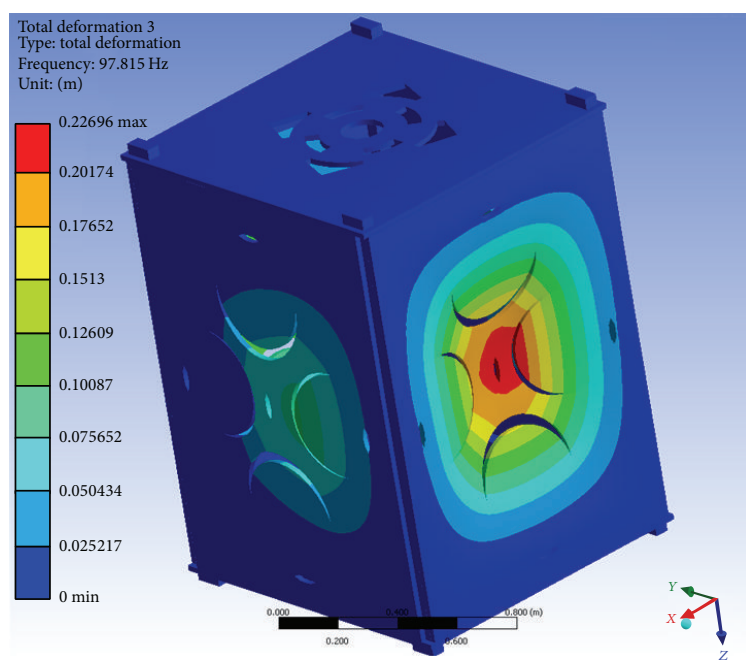

(c) Mode 3: Freq. $=97.82 \mathrm{~Hz}$ and Def. $=0.2269 \mathrm{~m}$

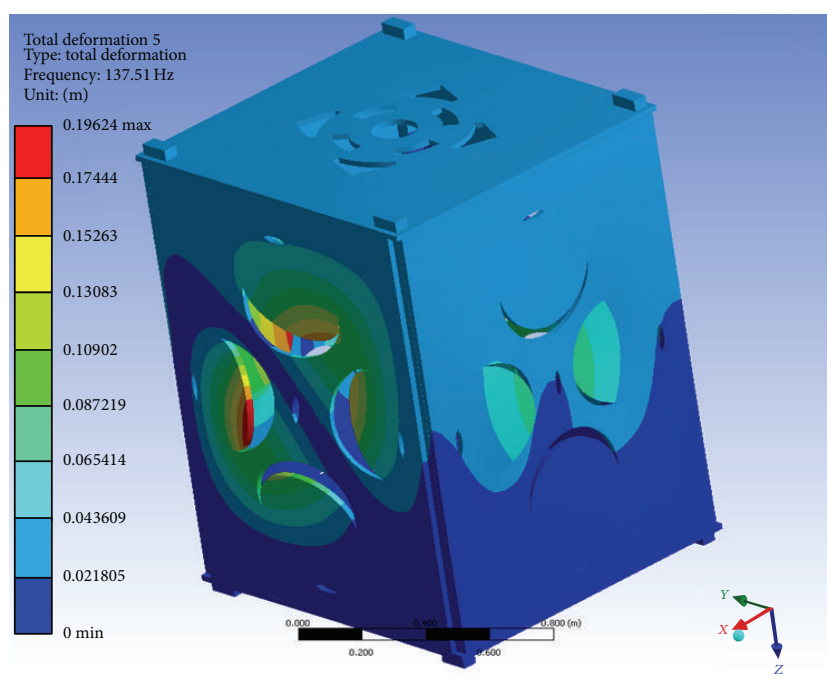

(e) Mode 5: Freq. $=137.51 \mathrm{~Hz}$ and Def. $=0.1962 \mathrm{~m}$

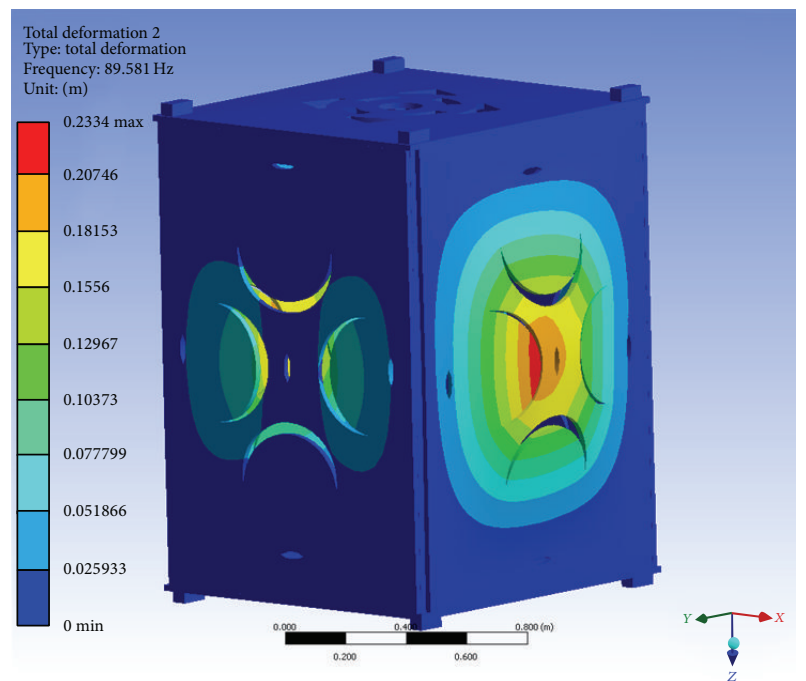

(b) Mode 2: Freq. $=89.58 \mathrm{~Hz}$ and Def. $=0.2334 \mathrm{~m}$

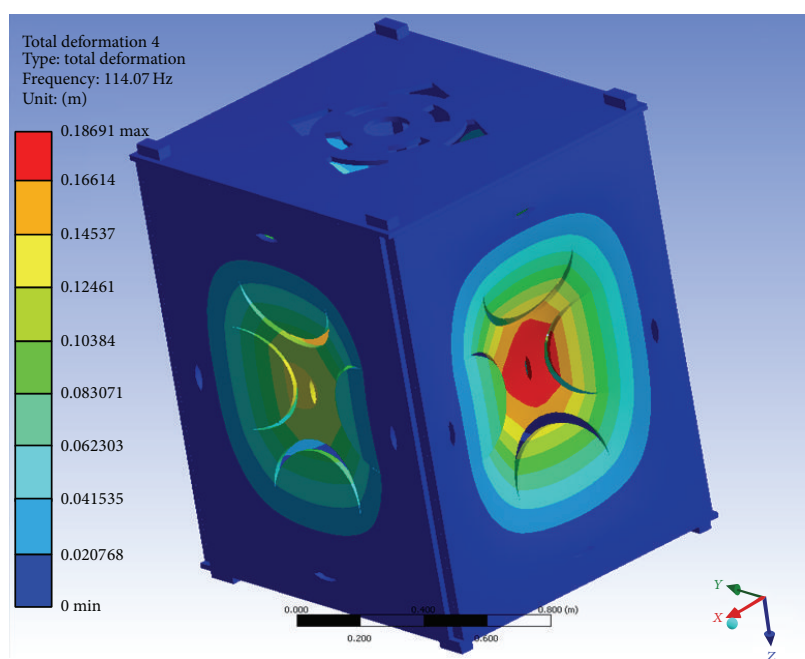

(d) Mode 4: Freq. $=114.07 \mathrm{~Hz}$ and Def. $=0.1869 \mathrm{~m}$

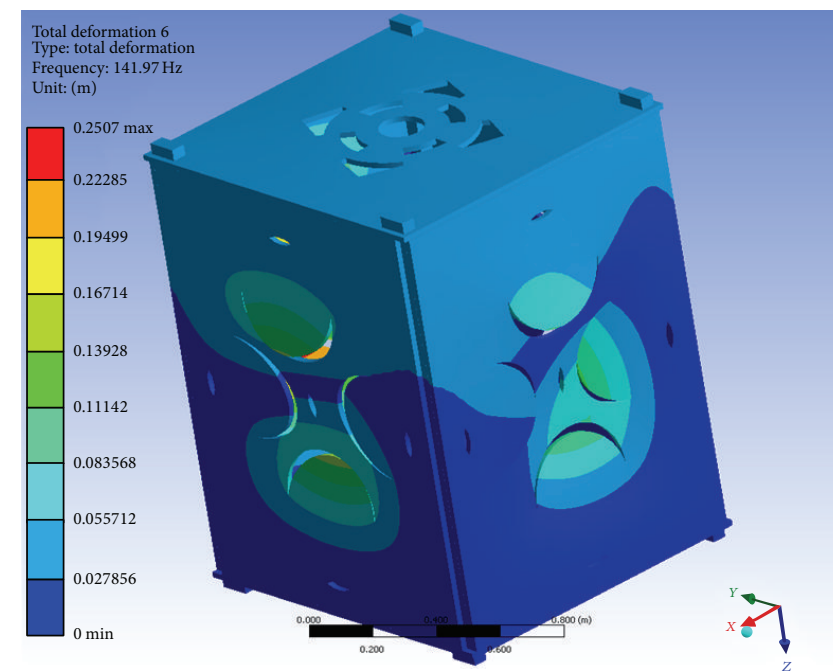

(f) Mode 6: Freq. $=141.97 \mathrm{~Hz}$ and Def. $=0.2507 \mathrm{~m}$

Figure 3: Continued. 


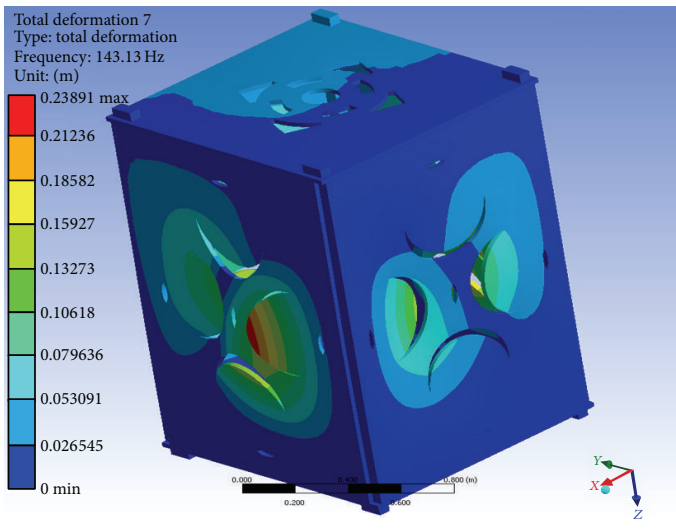

(g) Mode 7: Freq. $=143.13 \mathrm{~Hz}$ and Def. $=0.2389 \mathrm{~m}$

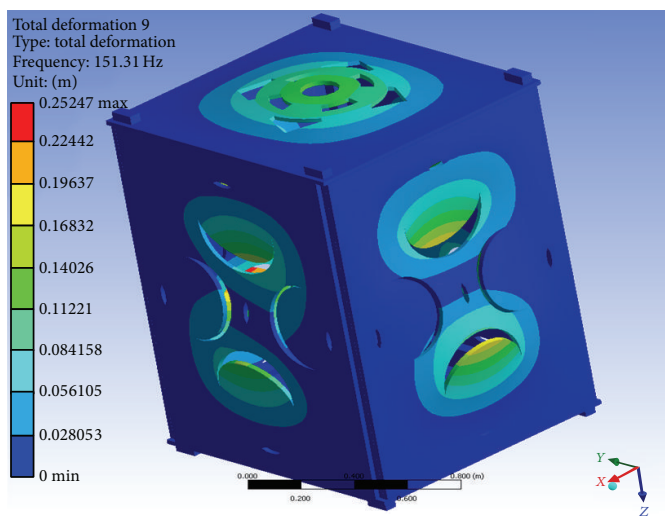

(i) Mode 9: Freq. $=151.31 \mathrm{~Hz}$ and Def. $=0.2524 \mathrm{~m}$

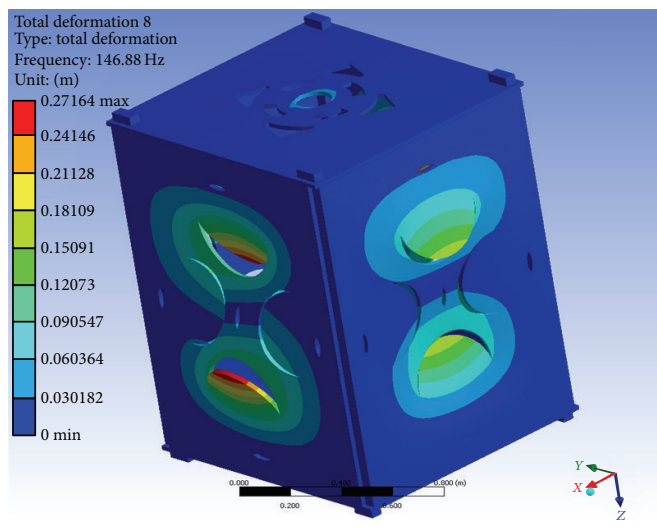

(h) Mode 8: Freq. $=146.88 \mathrm{~Hz}$ and Def. $=0.2716 \mathrm{~m}$

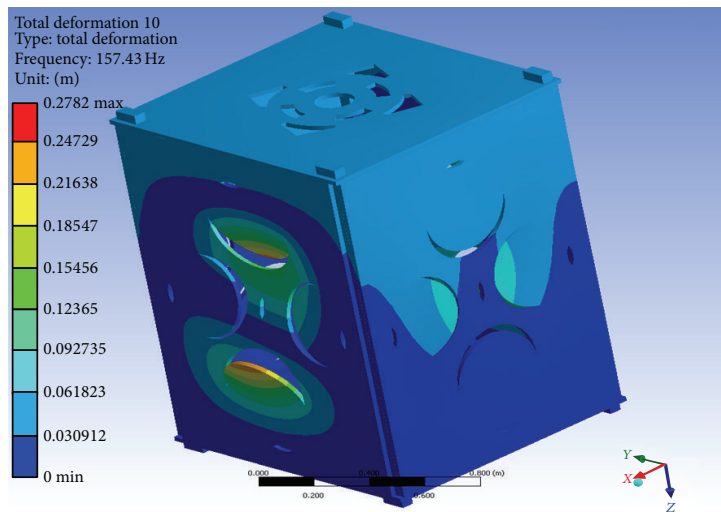

(j) Mode 10: Freq. $=157.43 \mathrm{~Hz}$ and Def. $=0.2782 \mathrm{~m}$

FIgURE 3: Ten modal shapes for different frequency values.

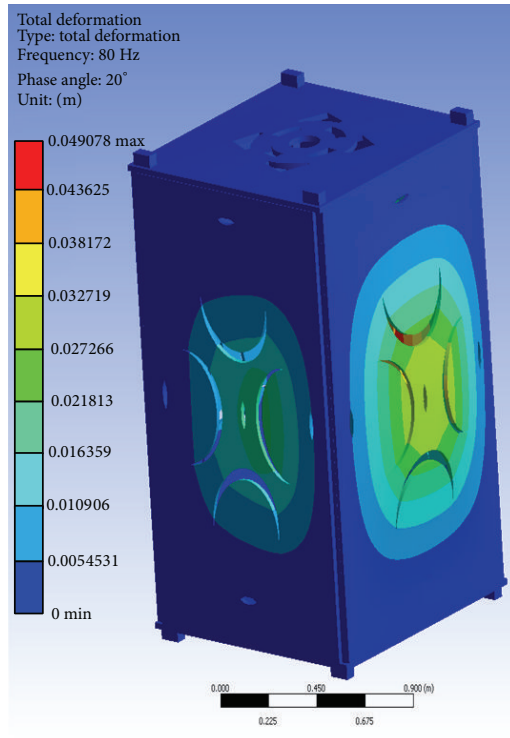

(a) Lateral direction

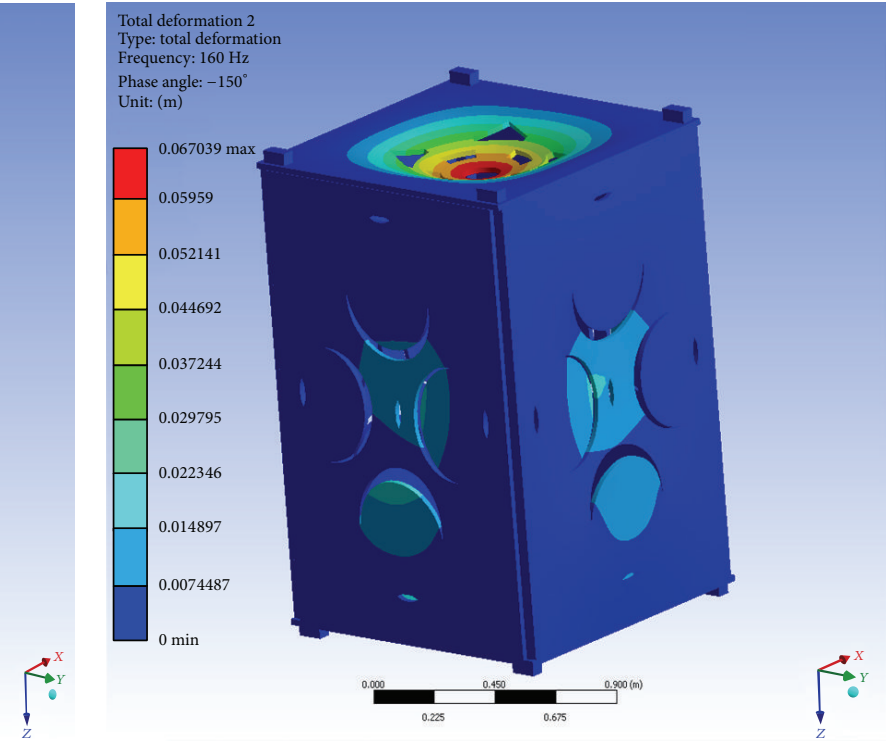

(b) Longitudinal direction

FIGURE 4: Deformation pattern. 


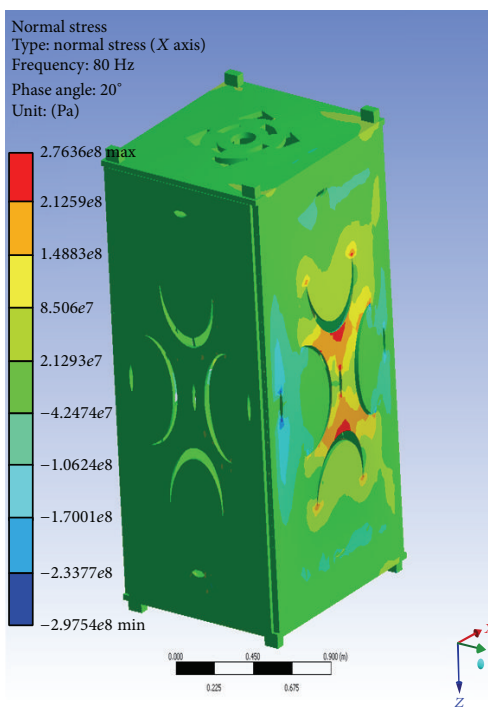

(a) Lateral direction

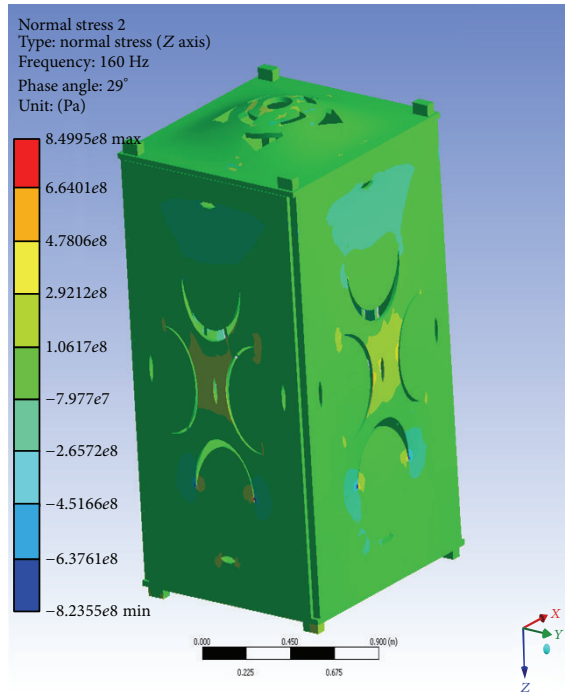

(b) Longitudinal direction

FIGURE 5: Stress pattern.

\section{Acknowledgments}

The author is cordially thankful to Mr. Hamid Khan for his devoted efforts and the reviewers for their valuable inputs and suggestions in finalizing this paper.

\section{References}

[1] A. Diana, J. Benjamin, and R. Guillaume, Design of a SwissCube Structure and Configuration, LMAF, EPFL, Lausanne, Switzerland, 2006.

[2] E. Pacheco, R. Conte, F. J. Mendieta, R. Muraoka, J. L. Medina, and A. Arvizu, "The SATEX project: a Mexican effort. The development of a micro-satellite patform for space technologies knowledge and human resources preparation," in Proceedings of the IEEE Aerospace Conference, pp. 677-686, March 2003.

[3] M. Sedighi and B. Mohammadi, On the Static and Dynamic Analysis of Small Satellite (Mesbah), IROST, Tehran, Iran, 2003.

[4] S. Ontaç, S. Dağ, and M. I. Gökler, "Structural finite element analysis of stiffened and honeycomb panels of the RASAT satellite," in Proceedings of the 3rd International Conference on Recent Advances in Space Technologies (RAST '07), pp. 171-175, Istanbul, Turkey, June 2007.

[5] B. Fufa, C. Zhao-Bo, and M. Wensheng, "Modeling and simulation of satellite solar panel deployment and locking," Information Technology Journal, vol. 9, no. 3, pp. 600-604, 2010.

[6] S. Libin and F. Hui, Static and Modal Analysis of Telescope Frame in Satellite, Tsinghua University and University of Science and Technology, Beijing, China, 2002.

[7] M. Cihan, O. O. Haktanir, I. Akbulut, and A. R. Aslan, Flight Dynamic Analysis of ITUpSAT1, Istanbul Technical University, İstanbul, Turkey, 2007.

[8] E. Braegen, D. Hayward, G. Hynd, and A. Thomas, Design, Build and Launch of a Small Satellite Based on CubeSat Designs, The University of Adelaide, Adelaide, Australia, 2007.
[9] H. Murtaza, "Designing a small satellite in LEO for remote sensing application," Journal of Space Technology, vol. 1, no. 1, pp. 11-16, 2011.

[10] B. D. Tapley, B. E. Schutz, and R. J. Eanes, "Satellite laser ranging and its applications," Celestial Mechanics, vol. 37, no. 3, pp. 247$261,1985$.

[11] L. Iorio, "An alternative derivation of the lense-thrilling drag on the orbit of a test body," IL Nuovo Cimento, vol. 116, no. 7, pp. 777-789, 2001

[12] L. Iorio, "An assessment of the systematic uncertainty in present and future tests of the lense-thirring effect with satellite laser ranging," Space Science Reviews, vol. 148, no. 1-4, pp. 363-381, 2009.

[13] G. Renzetti, "Are higher degree even zonals really harmful for the LARES/LAGEOS frame-dragging experiment?" Canadian Journal of Physics, vol. 90, no. 9, pp. 883-888, 2012.

[14] G. Renzetti, "History of the attempts to measure orbital framedragging with artificial satellites," Central European Journal of Physics, vol. 11, no. 5, pp. 531-544, 2013.

[15] J. J. Degnan, Millimeter Accuracy Satellite Laser Ranging: A Review, Code 920.1/Space Geodesy and Altimetry, NASA, Washington, DC, USA, 2013. 

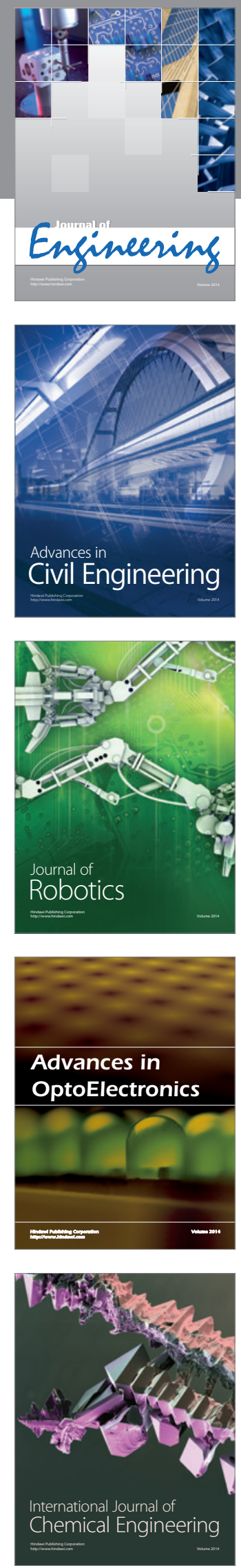

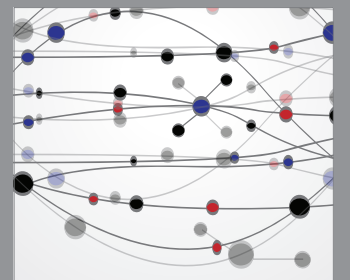

The Scientific World Journal
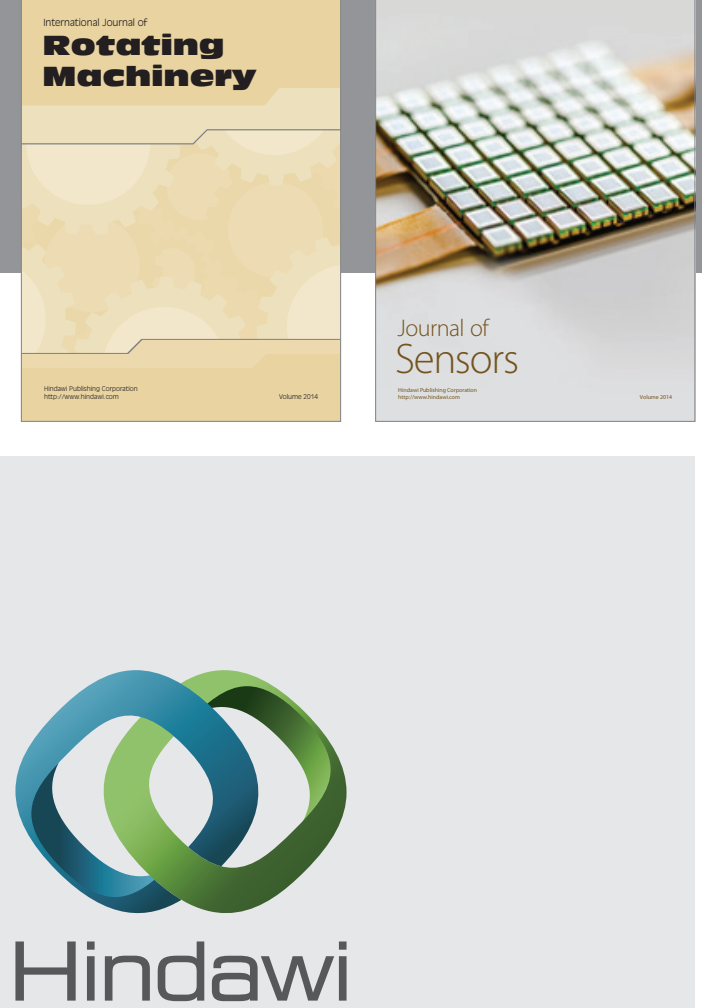

Submit your manuscripts at http://www.hindawi.com
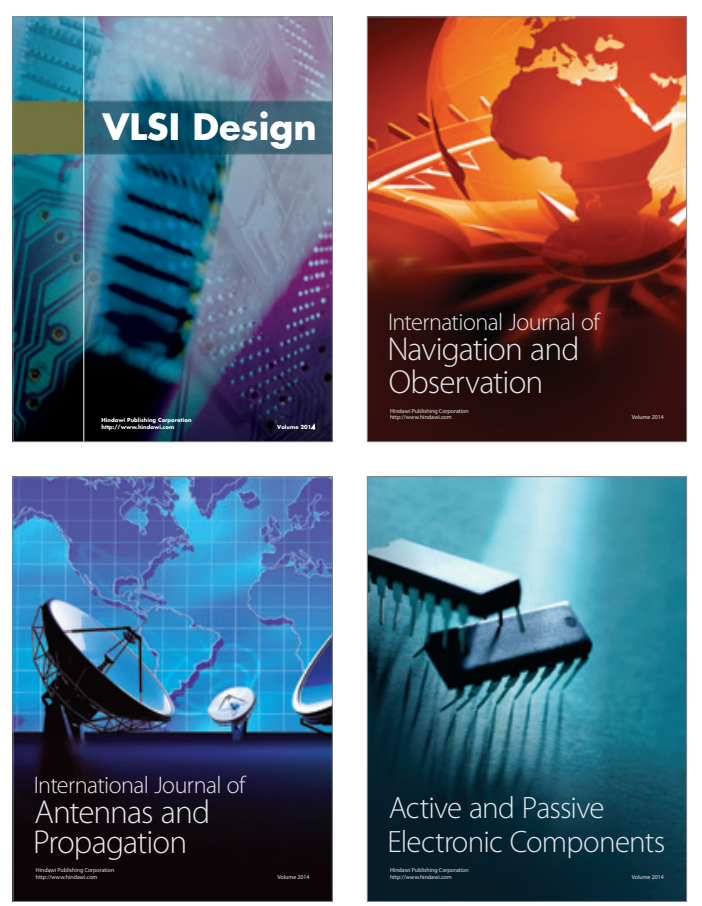
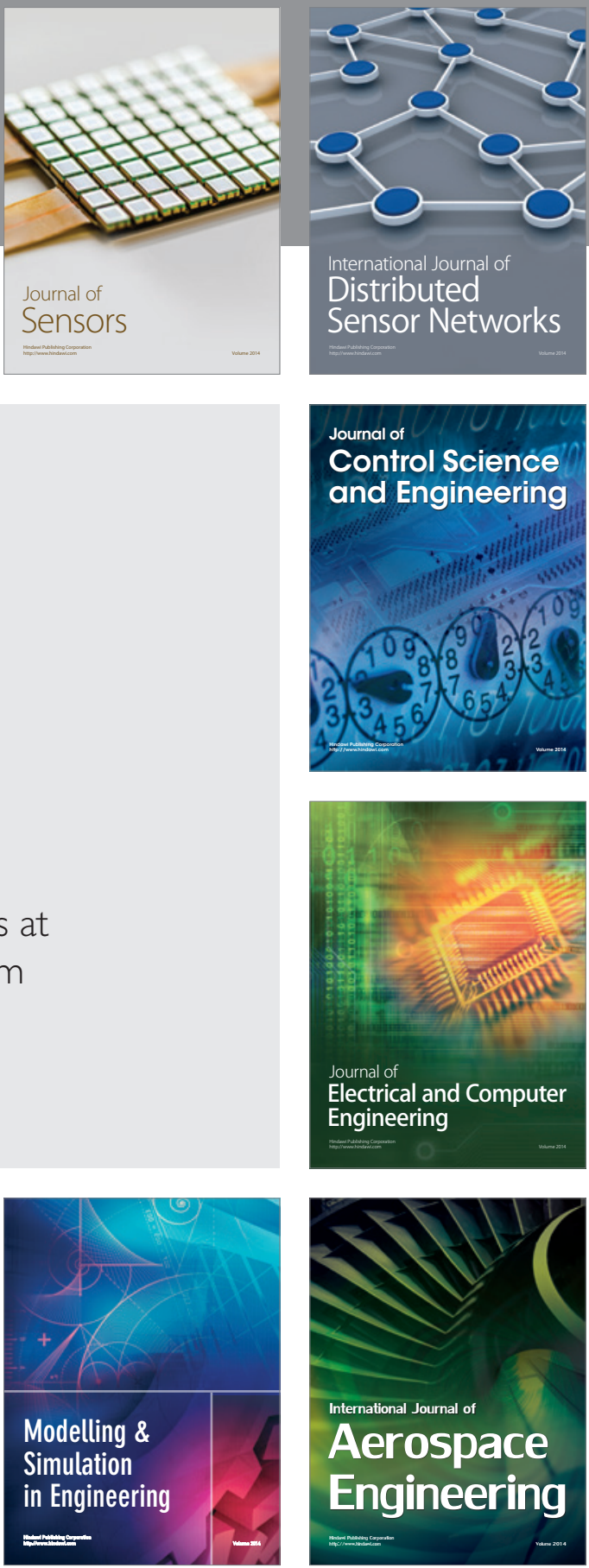

Journal of

Control Science

and Engineering
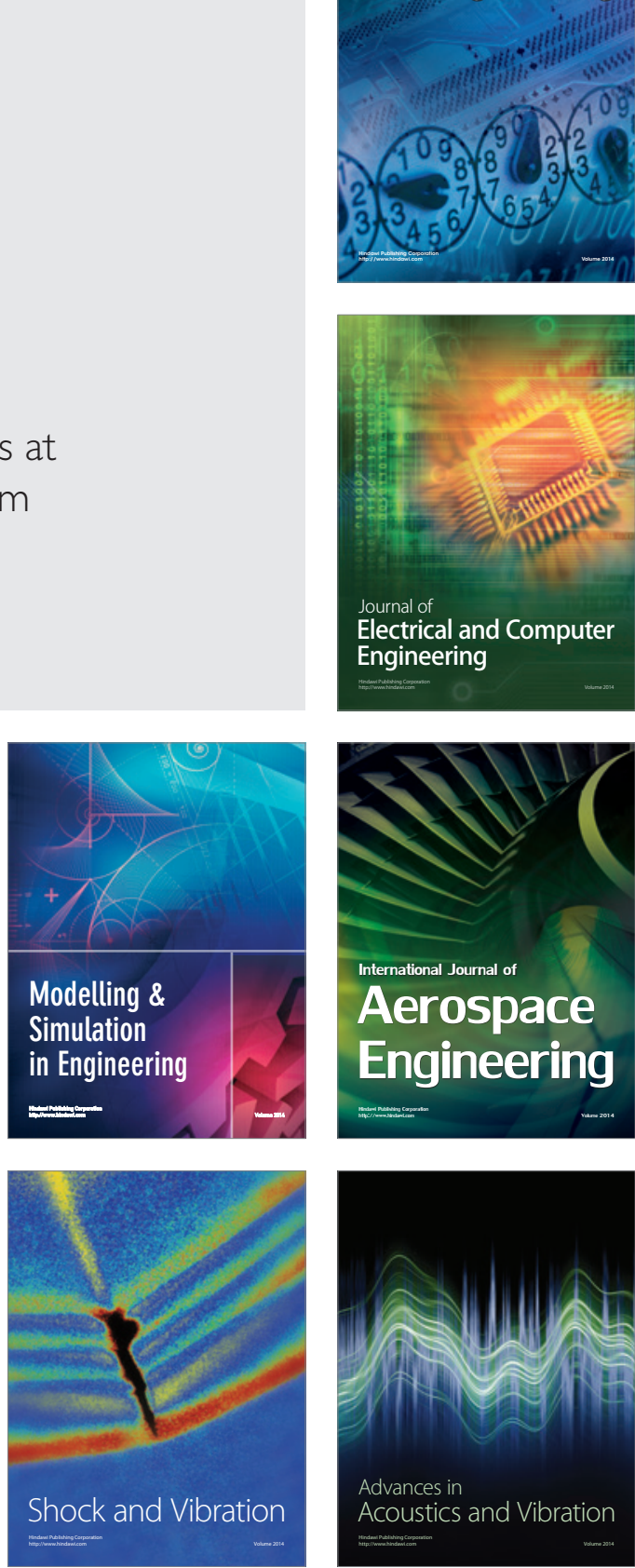